\title{
ON GENERALIZED K-UNIFORMLY CLOSE-TO-CONVEX FUNCTIONS OF JANOWSKI TYPE
}

\author{
AFIS SALIU ${ }^{1,2, *}$ \\ ${ }^{1}$ Department of Mathematics, COMSATS University, Chak Shahzad, Islamabad, 44000, Pakistan \\ ${ }^{2}$ Department of Mathematics, Gombe State University, Gombe State, Nigeria
}

*Corresponding author: afissaliu@gsu.edu.ng

\begin{abstract}
This work is concerned with the class of analytic functions that maps open unit disk onto conic domains. Necessary condition, arc length, growth rate of coefficients, radius problems and property of some integral transformation under the class are examined.
\end{abstract}

\section{INTRODUCTION}

Let $\mathcal{A}$ be the class of functions $f(z)$ whose series representation is given by

$$
f(z)=z+\sum_{n=2}^{\infty} a_{n} z^{n}
$$

and regular in an open unit disk $\Delta=\{z:|z|<1\}$. Let $\mathcal{S}$ denotes the class of univalent functions in $\Delta$ and $\mathcal{C}(\rho), \mathcal{S}^{*}(\rho)$ and $\mathcal{K}(\rho), 0 \leq \rho<1$ be the well known subclasses of $\mathcal{S}$ which consist of convex, starlike and close-to-convex functions of order $\rho$ respectively. $C(0) \equiv C, \mathcal{S}^{*}(0) \equiv \mathcal{S}^{*}$ and $\mathcal{K}(0) \equiv \mathcal{K}$ are the classes of convex, starlike and close-to-convex functions in $\Delta$ respectively. A function $f \in \mathcal{A}$ is subordinate to $g \in \mathcal{A}$ (written as $f(z) \prec g(z))$ if there exists a function $w(z)$ with $|w(z)|<1$ and $w(0)=0$ such that $f(z)=g(w(z))$. In addition, if $g(z)$ is univalent in $\Delta$, then $f(0)=g(0)$ and $f(\Delta) \subset g(\Delta)$ [4].

2010 Mathematics Subject Classification. 30C45, 30C50, 30C55.

Key words and phrases. Analytic functions; Janowski functions; Conic domains.

(C)2019 Authors retain the copyrights of their papers, and all open access articles are distributed under the terms of the Creative Commons Attribution License. 
Let $\mathcal{H}$ be the class of functions $p(z)=1+\sum_{n=1}^{\infty} c_{n} z^{n}$ that are regular in $\Delta$ with $p(0)=1$. Then $p \in$ $P[A, B],-1 \leq B<A \leq 1$ if and only if $p(z) \prec \frac{1+A z}{1+B z}$, or equivalently

$$
p(z)=\frac{(A+1) h(z)-(A-1)}{(B+1) h(z)-(B-1)}
$$

where $h \in \mathcal{P}[1,-1]=\mathcal{P}$, the class of functions with positive real part. This class of functions was first considered and study extensively by Janowski [6].

Kanas and Wisniowska $[7,8]$ introduced the class of $k$-uniformly convex functions and $k$-uniformly starlike function denoted by $k-U C V$ and $k-U S T$ respectively, which were defined subject to the conic domain $\Omega_{k}, k \geq 0$, given by

$$
\Omega_{k}=\left\{u+i v: u>k \sqrt{(u-1)^{2}+v^{2}}\right\}
$$

This domain represents the right half plane for $k=0$, the right branch of hyperbola for $0<k<1$ and an ellipse when $k>1$. The function $p_{k}(z)$ plays an extremal role for all functions that maps $\Delta$ onto $\Omega_{k}$ and it is given by

$$
p_{k}(z)=\left\{\begin{array}{l}
\frac{1+z}{1-z}, k=0 \\
1+\frac{2}{\pi^{2}}\left(\log \frac{1+\sqrt{z}}{1-\sqrt{z}}\right)^{2}, k=1 \\
1+\frac{2}{1-k^{2}} \sinh ^{2}\left[\left(\frac{2}{\pi} \arccos k\right) \arctan \sqrt{z}\right], 0<k<1 \\
1+\frac{1}{1-k^{2}} \sin \left[\frac{\pi}{2 R(t)} \int_{0}^{\frac{u(z)}{\sqrt{t}}} \frac{1}{\sqrt{1-x^{2}} \sqrt{1-(t x)^{2}}} d x\right]+\frac{1}{k^{2}-1}, k>1,
\end{array}\right.
$$

where $u(z)=\frac{z-\sqrt{t}}{1-\sqrt{t z}}, t \in(0,1), z \in \Delta$ and $t$ is chosen such that $k=\cosh \left(\frac{\pi R^{\prime}(t)}{4 R(t)}\right), R(t)$ is Legendre's complete elliptic integral of the first kind and $R^{\prime}(t)$ is the complementary integral of $R(t)$ [7].

We denote by $P\left(p_{k}\right)$, the class of functions that are subordinate to $p_{k}(z)$. Ronning [20] proved that for $p \in P\left(p_{k}\right)$, there exists a function $h \in \mathcal{P}$ such that $p(z)=h^{\gamma}(z)$ and $\gamma$ is given as:

$$
\gamma=\frac{2}{\pi} \arctan \left(\frac{1}{k}\right)
$$

It was also shown in [9] that for $p_{k}(z)=1+\delta_{k} z+\cdots \in P\left(p_{k}\right)$,

$$
\delta_{k}=\left\{\begin{array}{l}
\frac{8(\arccos k)^{2}}{\pi^{2}\left(1-k^{2}\right)}, 0 \leq k<1, \\
\frac{8}{\pi^{2}}, k=1 \\
\frac{\pi^{2}}{4\left(k^{2}-1\right) \sqrt{t}(1+t) R^{2}(t)}, k>1 .
\end{array}\right.
$$

Very recently, K.I. Noor [18] extended the domain $\Omega_{k}$ to that of Janowski type, $\Omega_{k}[A, B]$, $-1 \leq B<A \leq 1$ and defined it as

$$
\Omega_{k}[A, B]=\left\{w: \operatorname{Re}\left(\frac{(B-1) h(z)-(A-1)}{(B+1) h(z)-(A+1}\right)>k\left|\frac{(B-1) h(z)-(A-1)}{(B+1) h(z)-(A+1)}-1\right|\right\}
$$


or alternatively,

$$
\begin{aligned}
\Omega_{k}[A, B] & =\left\{u+i v:\left[\left(B^{2}-1\right)\left(u^{2}+v^{2}\right)-2(A B-1) u+\left(A^{2}-1\right)\right]^{2}\right. \\
& \left.>k^{2}\left[\left(-2(B+1)\left(u^{2}+v^{2}\right)+2(A+B+2) u-2(A+1)\right)^{2}+4(A-B)^{2} v^{2}\right]\right\} .
\end{aligned}
$$

Geometrically, the effect of $\Omega_{k}[A, B]$ on $\Omega_{k}$ was described in [18].

We denote by $k-P[A, B],-1 \leq B<A \leq 1$, the class of functions that map $\Delta$ onto $\Omega_{k}[A, B]$. Equivalently, we say $p \in k-P[A, B]$ if and only if

$$
p(z) \prec \frac{(A+1) p_{k}(z)-(A-1)}{(B+1) p_{k}(z)-(B-1)}, k \geq 0,-1 \leq B<A \leq 1,
$$

where $p_{k}$ is given by (1.3). Also, it is worthy to note that $p \in k-P[A, B] \subset \mathcal{P}\left(\beta_{1}\right)$ which implies that $p(z)=\left(1-\beta_{1}\right) h_{1}(z)+\beta_{1}$, (see [18]) where $h_{1} \in \mathcal{P}$ and $\beta_{1}$ is given by

$$
\beta_{1}=\frac{2 k+1-A}{2 k+1-B}
$$

We extend the class $k-P[A, B]$ as follows:

Definition 1.1. Let $p(z) \in \mathcal{H}$. Then $p \in k-P_{\mu}[A, B]$ if and only if for $\mu \geq 2, k \geq 0,-1 \leq B<A \leq 1$, we have

$$
p(z)=\frac{\mu+2}{4} p_{1}(z)-\frac{\mu-2}{4} p_{2}(z), p_{1}, p_{2} \in k-P[A, B] .
$$

Definition 1.2. Let $p(z) \in \mathcal{H}$. Then $p \in k-P_{\mu}[A, B, \alpha]$ if and only if for $\mu \geq 2, k \geq 0$,

$-1 \leq B<A \leq 1, \alpha \in[0,1)$, we have

$$
p(z)=(1-\alpha) h(z)+\alpha, h(z) \in k-P_{\mu}[A, B] .
$$

For $k=0, A=1, B=-1, \alpha=0$, we have the class $P_{\mu}$ introduced and studied in [22]. Also, when $\mu=2, \alpha=0$, we get the class $k-P[A, B]$, which was first considered by K.I. Noor in [18]. The class $k-P_{\mu}\left[1-2 \gamma^{*},-1,0\right], \gamma^{*} \in[0,1)$ is the same as the class $k-P_{\mu}\left(\gamma^{*}\right)$ studied in [17].

We now define the following classes of functions.

Definition 1.3. Let $f \in \mathcal{A}$. Then $f \in k-U \mathcal{R}_{\mu}[A, B, \alpha], k \geq 0, \mu \geq 2, \alpha \in[0,1),-1 \leq B<A \leq 1$, if and only if

$$
\frac{z f^{\prime}(z)}{f(z)} \in k-P_{\mu}[A, B, \alpha]
$$

Definition 1.4. Let $f \in \mathcal{A}$. Then $f \in k-U \mathcal{V}_{\mu}[A, B, \alpha], k \geq 0, \mu \geq 2, \alpha \in[0,1),-1 \leq B<A \leq 1$, if and only if

$$
\frac{\left(z f^{\prime}(z)\right)^{\prime}}{f^{\prime}(z)} \in k-P_{\mu}[A, B, \alpha]
$$

It is obvious to note that $f \in k-U \mathcal{V}_{\mu}[A, B, \alpha] \Leftrightarrow z f^{\prime}(z) \in k-U \mathcal{R}_{\mu}[A, B, \alpha]$. Also, $0-U \mathcal{V}_{\mu}[1,-1,0]=\mathcal{V}_{\mu}$, is the class of functions of bounded boundary rotation(see [1], [22]) 
Definition 1.5. Let $f \in \mathcal{A}$. Then $f \in k-\mathcal{T}_{\mu}[A, B, C, D, \alpha], k \geq 0, \mu \geq 2, \alpha \in[0,1),-1 \leq B<A \leq 1,-1 \leq$ $D<C \leq 1$ if and only if there exists $g \in k-U \mathcal{R}_{\mu}[C, D, \alpha]$ such that

$$
\frac{z f^{\prime}(z)}{g(z)} \in k-P[A, B]
$$

or equivalently as

$$
\frac{f^{\prime}(z)}{g^{\prime}(z)} \in k-P[A, B]
$$

where $g \in k-U \mathcal{V}_{\mu}[C, D, \alpha]$.

We note the following special cases.

i $0-\mathcal{T}_{2}[A, B, C, D, 0]=\mathcal{K}[A, B, C, D]$, is the class of functions studied by Silvia [23].

ii $0-\mathcal{T}_{2}[1,-1,1,-1,0]=\mathcal{K}$, is the class of close to convex functions examined by Kaplan [10].

iii $k-\mathcal{T}_{2}[A, B, C, D, 0]=k-U \mathcal{K}[A, B, C, D]$, the class considered in [12]

iv $1-\mathcal{T}_{2}[1,-1,1,-1,0]=U C C$, is the class of uniformly close to convex functions explored by Kumar and Ramesha [11].

$\mathrm{v} 1-\mathcal{T}_{2}[1-2 \rho,-1,1,-1,0]=U C C(\rho)$ is the class of uniformly close to convex of order $\rho,-1 \leq \rho<1$ that was taken into account in [24].

vi $0-\mathcal{T}_{\mu}[1-2 \rho,-1,1-2 \rho,-1,0]=\mathcal{T}_{\mu}(\rho), \rho \in[0,1)$, is the class of functions that was studied by K.I.

Noor in $[16]$

vii $0-\mathcal{T}_{\mu}[1,-1,1,-1,0]=\mathcal{T}_{\mu}$, is the class of generalized close-to-convex functions introduced and studied in [15].

Let

$$
G(a, b, c ; z)=\frac{\Gamma(c)}{\Gamma(a) \Gamma(c-a)} \int_{0}^{1} u^{a-1}(1-u)^{c-a-1}(1-z u)^{-b} d u,
$$

where $\operatorname{Re} a>0, \operatorname{Re}(c-a)>0, \Gamma$ denotes the Gamma function and $G(a, b, c ; z)$ is hypergeometric function.

Unless if otherwise stated, we lay down once and for all that $k \geq 0, \mu \geq 2, \alpha \in[0,1),-1 \leq B<A \leq$ $1,-1 \leq D<C \leq 1$.

\section{Some Preliminary Lemmas}

We need the following lemmas.

Lemma 2.1. [1] Every function $f \in \mathcal{V}_{\mu}$ is a close to convex function of order $\frac{\mu}{2}-1$.

Lemma 2.2. [13] Let $p \in P[A, B]$. Then

$$
|\arg p(z)| \leq \sin ^{-1} \frac{(A-B) r}{1-A B r^{2}}
$$


Lemma 2.3. [5] Let $p(z) \in \mathcal{H}$ with $\operatorname{Rep}(z)>0, z=r e^{i \theta}(0<r<1)$. Then

$$
\int_{0}^{2 \pi}\left|p\left(r e^{i \theta}\right)\right|^{\lambda} d \theta<C(\lambda) \frac{1}{(1-r)^{\lambda-1}},
$$

where $C(\lambda)$ is a constant and $\lambda>1$.

Lemma 2.4. [1] Let $g \in \mathcal{V}_{\mu}$. Then for $\mu>3$ there exists $s_{1} \in \mathcal{S}^{*}(0)$ and $p \in \mathcal{P}$ such that

$$
z g^{\prime}(z)=s_{1}(z)(p(z))^{\frac{\mu}{2}-1}
$$

Lemma 2.5. [19] Let $q(z)$ be analytic in $\Delta$ with $q(0)=1$. If $\varrho \geq 1, \operatorname{Rec} \geq 0,0 \leq \theta_{1}<\theta_{2} \leq 2 \pi, z=r e^{i \theta}$, then

$$
\int_{\theta_{1}}^{\theta_{2}} \operatorname{Re}\left\{q(z)+\frac{\varrho z q^{\prime}(z)}{c \varrho+q(z)}\right\} d \theta>-\sigma \pi, \quad(0<\sigma \leq 1),
$$

implies

$$
\int_{\theta_{1}}^{\theta_{2}} \operatorname{Req}(z) d \theta>-\sigma \pi
$$

\section{Main Result}

In this section, we present our main work.

Theorem 3.1. Let $f \in k-U \mathcal{V}_{\mu}[C, D, \alpha]$. Then $f^{\prime}(z)=\left(\phi^{\prime}(z)\right)^{1-\beta}$, where $\phi \in \mathcal{V}_{\mu}, \beta=\alpha+(1-\alpha) \beta_{1}$ and $\beta_{1}$ is given by (1.9).

Proof. Let $f \in k-U \mathcal{V}_{\mu}[C, D, \alpha]$. Then

$$
\frac{\left(z f^{\prime}(z)\right)^{\prime}}{f^{\prime}(z)}=(1-\alpha)\left[\frac{\mu+2}{4} p_{1}(z)-\frac{\mu-2}{4} p_{2}(z)\right]+\alpha, p_{1}, p_{2} \in k-P[C, D] \subset \mathcal{P}\left(\beta_{2}\right) .
$$

where $\beta_{2}=\frac{2 k+1-C}{2 k+1-D}$. Therefore,

$$
\frac{\left(z f^{\prime}(z)\right)^{\prime}}{f^{\prime}(z)}=\frac{\mu+2}{4} h_{1}(z)-\frac{\mu-2}{4} h_{2}(z), h_{1}, h_{2} \in \mathcal{P}(\beta)
$$

where

$$
\beta=(1-\alpha) \beta_{2}+\alpha=\frac{2 k+1-C+\alpha(C-D)}{2 k+1-D} .
$$

Thus, there exist $f_{1}, f_{2} \in C(\beta)$ such that

$$
\frac{\left(z f^{\prime}(z)\right)^{\prime}}{f^{\prime}(z)}=\frac{\mu+2}{4} \frac{\left(z f_{1}^{\prime}(z)\right)^{\prime}}{f_{1}^{\prime}(z)}-\frac{\mu-2}{4} \frac{\left(z f_{2}^{\prime}(z)\right)^{\prime}}{f_{2}^{\prime}(z)}
$$


Integrating and using the result due to Brannan [1], we have

$$
\begin{aligned}
f^{\prime}(z) & =\frac{\left(f_{1}^{\prime}(z)\right)^{\left(\frac{\mu+2}{4}\right)}}{\left(f_{2}^{\prime}(z)\right)^{\left(\frac{\mu-2}{4}\right)}} \\
& =\varphi^{\prime}(z), \varphi \in \mathcal{V}_{\mu}(\beta) \\
& =\left(\phi^{\prime}(z)\right)^{1-\beta}, \phi \in \mathcal{V}_{\mu}
\end{aligned}
$$

Theorem 3.2. Let $f \in k-\mathcal{T}_{\mu}[A, B, C, D, \alpha]$. Then for $\mu>2,0 \leq \theta_{1}<\theta_{2} \leq 2 \pi, z=r e^{i \theta}$,

$$
\int_{\theta_{1}}^{\theta_{2}} \operatorname{Re} \frac{\left(z f^{\prime}(z)\right)^{\prime}}{f^{\prime}(z)} d \theta>-\pi\left[(1-\alpha)\left(\frac{C-D}{2 k+1-D}\right)\left(\frac{\mu}{2}-1\right)+\gamma\right]
$$

where $\gamma$ is given by (1.4).

Proof. Let

$$
f^{\prime}(z)=g^{\prime}(z) h(z)
$$

where $g \in k-U \mathcal{V}_{\mu}[C, D, \alpha], h \in k-P[A, B]$. From Theorem 3.1, we write

$$
z g^{\prime}(z)=z^{\beta}\left(z \phi^{\prime}(z)\right)^{1-\beta}
$$

By logarithmic differentiation and using Lemma 2.1, it follows that

$$
\begin{aligned}
\int_{\theta_{1}}^{\theta_{2}} \frac{\left(z g^{\prime}(z)\right)^{\prime}}{g^{\prime}(z)} d \theta & =\beta\left(\theta_{2}-\theta_{1}\right)+(1-\beta) \int_{\theta_{1}}^{\theta_{2}} \frac{\left(z \phi^{\prime}(z)\right)^{\prime}}{\phi^{\prime}(z)} d \theta \\
& >-\pi(1-\beta)\left(\frac{\mu}{2}-1\right) .
\end{aligned}
$$

Since $h \in k-P[A, B]$, there exists $h_{1} \in P[A, B]$ such that $h(z)=\left(h_{1}(z)\right)^{\gamma}$, where $\gamma$ is given by (1.4). Therefore,

$$
f^{\prime}(z)=g^{\prime}(z)\left(h_{1}(z)\right)^{\gamma}
$$

and thus,

$$
\int_{\theta_{1}}^{\theta_{2}} \operatorname{Re} \frac{\left(r e^{i \theta} f^{\prime}\left(r e^{i \theta}\right)\right)^{\prime}}{f^{\prime}\left(r e^{i \theta}\right)} d \theta=\int_{\theta_{1}}^{\theta_{2}} \operatorname{Re} \frac{\left(r e^{i \theta} g^{\prime}\left(r e^{i \theta}\right)\right)^{\prime}}{g^{\prime}\left(r e^{i \theta}\right)} d \theta+\gamma \int_{\theta_{1}}^{\theta_{2}} R e \frac{r e^{i \theta} h_{1}^{\prime}\left(r e^{i \theta}\right)}{h_{1}\left(r e^{i \theta}\right)} d \theta
$$

But

$$
\int_{\theta_{1}}^{\theta_{2}} R e \frac{r e^{i \theta} h_{1}^{\prime}\left(r e^{i \theta}\right)}{h_{1}\left(r e^{i \theta}\right)} d \theta=\arg h_{1}\left(r e^{i \theta_{2}}\right)-\arg h_{1}\left(r e^{i \theta_{1}}\right)
$$

which implies

$$
\max _{h_{1} \in P[A, B]}\left|\int_{\theta_{1}}^{\theta_{2}} R e \frac{r e^{i \theta} h_{1}^{\prime}\left(r e^{i \theta}\right)}{h_{1}\left(r e^{i \theta}\right)} d \theta\right| \leq \max _{h_{1} \in P[A, B]}\left|\arg h_{1}\left(r e^{i \theta_{2}}\right)\right|+\max _{h_{1} \in P[A, B]}\left|\arg h_{1}\left(r e^{i \theta_{1}}\right)\right| .
$$


Using Lemma 2.2 in (3.5), we get

$$
\max _{h_{1} \in P[A, B]}\left|\int_{\theta_{1}}^{\theta_{2}} R e \frac{r e^{i \theta} h_{1}^{\prime}\left(r e^{i \theta}\right)}{h_{1}\left(r e^{i \theta}\right)} d \theta\right| \leq \pi-2 \cos ^{-1} \frac{(A-B) r}{1-A B r^{2}} .
$$

This means that

$$
\int_{\theta_{1}}^{\theta_{2}} \operatorname{Re} \frac{r e^{i \theta} h_{1}^{\prime}\left(r e^{i \theta}\right)}{h_{1}\left(r e^{i \theta}\right)} d \theta \geq-\pi
$$

Using (3.3) and (3.6) in (3.4), we have the result and this completes the proof.

Corollary 3.1. [14] Let $f \in k-\mathcal{T}_{\mu}[1,-1,1,-1,0]$. Then for $\mu>2,0 \leq \theta_{1}<\theta_{2} \leq 2 \pi, z=r e^{i \theta}$,

$$
\int_{\theta_{1}}^{\theta_{2}} \operatorname{Re} \frac{\left(z f^{\prime}(z)\right)^{\prime}}{f^{\prime}(z)} d \theta>-\pi\left(\frac{\mu-2}{2(k+1)}+\gamma\right) .
$$

Corollary 3.2. Let $f \in 1-\mathcal{T}_{\mu}[1,-1,1,-1,0]$. Then for $\mu>2,0 \leq \theta_{1}<\theta_{2} \leq 2 \pi, z=r e^{i \theta}$,

$$
\int_{\theta_{1}}^{\theta_{2}} R e \frac{\left(z f^{\prime}(z)\right)^{\prime}}{f^{\prime}(z)} d \theta>-\pi \frac{\mu}{4}
$$

Corollary 3.3. [15] Let $f \in 0-\mathcal{T}_{\mu}[1,-1,1,-1,0]$. Then for $0 \leq \theta_{1}<\theta_{2} \leq 2 \pi, z=r e^{i \theta}$,

$$
\int_{\theta_{1}}^{\theta_{2}} \operatorname{Re} \frac{\left(z f^{\prime}(z)\right)^{\prime}}{f^{\prime}(z)} d \theta>-\pi \frac{\mu}{2}
$$

Using Goodman result in [3], we have the following.

Corollary 3.4. The function $f \in k-\mathcal{T}_{\mu}[A, B, C, D, \alpha]$ is univalent in $\Delta$ for $\mu<2\left(\frac{1-\gamma}{1-\alpha}\right)\left(\frac{2 k+1-D}{C-D}\right)+2$.

Remark 3.1. Let $f \in k-\mathcal{T}_{\mu}[A, B, C, D, \alpha]$. Then setting $\frac{z f^{\prime}(z)}{f(z)}=p(z)$ in Theorem 3.2 and applying Lemma 2.5, we have

$$
\int_{\theta_{1}}^{\theta_{2}} R e \frac{z f^{\prime}(z)}{f(z)} d \theta>-\pi\left[(1-\alpha)\left(\frac{C-D}{2 k+1-D}\right)\left(\frac{\mu}{2}-1\right)+\gamma\right] .
$$

Theorem 3.3. Let $f \in k-\mathcal{T}_{\mu}[A, B, C, D, \alpha]$. Then

$$
\left|\arg f^{\prime}(z)\right| \leq(1-\beta) \mu \sin ^{-1} r+\gamma \sin ^{-1} \frac{(A-B) r}{1-A B r^{2}}
$$

where $\gamma$ is given by (1.4).

Proof. Let

$$
f^{\prime}(z)=\left(\phi^{\prime}(z)\right)^{1-\beta}(h(z))^{\gamma}, \phi \in \mathcal{V}_{\mu}, h \in P[A, B]
$$


where $\beta$ is given by (3.1). Therefore,

$$
\left|\arg f^{\prime}(z)\right| \leq(1-\beta)\left|\arg \left(\phi^{\prime}(z)\right)\right|\left|\arg (h(z))^{\gamma}\right| .
$$

Since it is well known in [21], that for $\phi \in \mathcal{V}_{\mu},\left|\arg \left(\phi^{\prime}(z)\right)\right| \leq \mu \sin ^{-1} r$. Using this results and Lemma 2.2, the proof is complete.

Theorem 3.4. Let $p(z)=1+\sum_{n=1}^{\infty} c_{n} z^{n} \in k-P_{\mu}[A, B, \alpha]$. Then for $n \geq 1$,

$$
\left|c_{n}\right| \leq \frac{\mu(1-\alpha)(A-B)\left|\delta_{k}\right|}{4}
$$

where $\delta_{k}$ is given by (1.5).

Proof. Since $p \in k-P_{\mu}[A, B, \alpha]$, then we write

$$
p(z)=\frac{\mu+2}{4}\left[(1-\alpha) h_{1}(z)+\alpha\right]-\frac{\mu-2}{4}\left[(1-\alpha) h_{2}(z)+\alpha\right], h_{1}, h_{2} \in k-P[A, B] .
$$

Let $h_{1}(z)=1+\sum_{n=1}^{\infty} a_{n} z^{n}$ and $h_{2}(z)=1+\sum_{n=1}^{\infty} b_{n} z^{n}$. Then from (3.7), we have

$$
1+\sum_{n=1}^{\infty} c_{n} z^{n}=1+\sum_{n=1}^{\infty}\left[\frac{\mu+2}{4} a_{n}-\frac{\mu-2}{4} b_{n}\right] z^{n} .
$$

Comparing the coefficient of $z^{n}$, we obtain

$$
\left|c_{n}\right| \leq(1-\alpha)\left[\frac{\mu+2}{4}\left|a_{n}\right|+\frac{\mu-2}{4}\left|b_{n}\right|\right] .
$$

It has been established in [18] that for $h(z)=1+\sum_{n=1}^{\infty} d_{n} z^{n} \in k-P[A, B]$,

$$
\left|d_{n}\right| \leq \frac{(A-B)\left|\delta_{k}\right|}{2}, n \geq 1
$$

and $\delta_{k}$ is given by (1.5). Using this result, it follows that

$$
\left|c_{n}\right| \leq \frac{\mu(1-\alpha)(A-B)\left|\delta_{k}\right|}{4}
$$

and this completes the proof.

Corollary 3.5. Let $p(z)=1+\sum_{n=1}^{\infty} c_{n} z^{n} \in 1-P_{2}[1,-1,0]$. Then for $n \geq 1$,

$$
\left|c_{n}\right| \leq \frac{8}{\pi^{2}}
$$

Corollary 3.6. [13] Let $p(z)=1+\sum_{n=1}^{\infty} c_{n} z^{n} \in 0-P_{2}[A, B, 0]$. Then for $n \geq 1$,

$$
\left|c_{n}\right| \leq A-B
$$

Corollary 3.7. [4] Let $p(z)=1+\sum_{n=1}^{\infty} c_{n} z^{n} \in 0-P_{2}[1,-1,0]$. Then for $n \geq 1$,

$$
\left|c_{n}\right| \leq 2
$$


Theorem 3.5. Let $p(z)=1+\sum_{n=1}^{\infty} c_{n} z^{n} \in k-P_{\mu}[A, B, \alpha]$. Then for $z=r e^{i \theta}(0<r<1)$,

$$
\frac{1}{2 \pi} \int_{0}^{2 \pi}|p(z)|^{2} d \theta \leq \frac{1+\left[\left(\frac{\mu(1-\alpha)(A-B)\left|\delta_{k}\right|}{4}\right)^{2}-1\right] r^{2}}{1-r^{2}} .
$$

Proof. Using Perseval's identity, we have

$$
\frac{1}{2 \pi} \int_{0}^{2 \pi}|p(z)|^{2} d \theta=1+\sum_{n=1}^{\infty}\left|c_{n}\right|^{2} r^{2 n} .
$$

Applying Theorem 3.4, we get

$$
\begin{aligned}
\frac{1}{2 \pi} \int_{0}^{2 \pi}|p(z)|^{2} d \theta & \leq 1+\left[\frac{\mu(1-\alpha)(A-B)\left|\delta_{k}\right|}{4}\right]^{2} \sum_{n=1}^{\infty} r^{2 n} \\
& =\frac{1+\left[\left(\frac{\mu(1-\alpha)(A-B)\left|\delta_{k}\right|}{4}\right)^{2}-1\right] r^{2}}{1-r^{2}} .
\end{aligned}
$$

Corollary 3.8. For $p \in 0-P_{2}[A, B, 0]=P[A, B]$,

$$
\frac{1}{2 \pi} \int_{0}^{2 \pi}|p(z)|^{2} d \theta \leq \frac{1+\left[(A-B)^{2}-1\right] r^{2}}{1-r^{2}} .
$$

Corollary 3.9. [16] For $p \in 0-P_{2}[1-2 \gamma,-1,0]=P(\gamma)$,

$$
\frac{1}{2 \pi} \int_{0}^{2 \pi}|p(z)|^{2} d \theta \leq \frac{1+\left[\mu^{2}(1-\gamma)^{2}-1\right] r^{2}}{1-r^{2}} .
$$

Theorem 3.6. [Arc Length Problem] Let $f \in k-\mathcal{T}_{\mu}[A, B, C, D, \alpha]$. Then for $\mu>\frac{2-\gamma}{1-\beta}+2$,

$$
\mathcal{L}_{r}(f) \leq C(\gamma, \beta, A, B)\left(\frac{1}{1-r}\right)^{(1-\beta)\left(\frac{\mu}{2}+1\right)+\gamma-1} \quad(r \rightarrow 1),
$$

where $C(\gamma, \beta, A, B)\left(\frac{1}{1-r}\right)$ is a constant that only depends on $\gamma, \beta, A, B$, where $\gamma$ and $\beta$ are respectively given by (1.4) and (3.1).

Proof. For $f \in k-\mathcal{T}_{\mu}[A, B, C, D, \alpha]$, and application of the result due to Brannan [1], we have

$$
z f^{\prime}(z)=z^{\beta}\left(s_{1}(z)\left(p_{1}(z)\right)^{\frac{\mu}{2}-1}\right)^{1-\beta}\left(h_{1}(z)\right)^{\gamma},
$$

where $s_{1} \in \mathcal{S}^{*}, p_{1} \in \mathcal{P}$ and $h_{1} \in P[A, B]$. Therefore, for $z=r e^{i \theta}(r<1)$,

$$
\mathcal{L}_{r}(f)=\int_{0}^{2 \pi}\left|z f^{\prime}(z)\right| d \theta
$$


Using (3.8), distortion theorem for starlike function $s_{1}(z)$, Hölder's inequality, Corollary 3.8 and Lemma 2.3, it follows that

$$
\begin{aligned}
\mathcal{L}_{r}(f) & \leq \frac{2 \pi}{(1-r)^{2(1-\beta)}}\left(\frac{1}{2 \pi} \int_{0}^{2 \pi}\left|p_{1}(z)\right|^{\frac{(1-\beta)(\mu-2)}{2-\gamma}} d \theta\right)^{\frac{2-\gamma}{2}}\left(\frac{1}{2 \pi} \int_{0}^{2 \pi}\left|h_{1}(z)\right|^{2} d \theta\right)^{\frac{\gamma}{2}} \\
& \leq \frac{2 \pi}{(1-r)^{2(1-\beta)}}\left(\frac{1+\left[(A-B)^{2}-1\right] r^{2}}{1-r^{2}}\right)^{\frac{\gamma}{2}}\left(\frac{1}{2 \pi} \int_{0}^{2 \pi}\left|p_{1}(z)\right|^{\frac{(1-\beta)(\mu-2)}{2-\gamma}} d \theta\right)^{\frac{2-\gamma}{2}} \\
& \leq C(\gamma, \beta, A, B, \alpha)\left(\frac{1}{1-r}\right)^{(1-\beta)\left(\frac{\mu}{2}+1\right)+\gamma-1} \quad(r \rightarrow 1),
\end{aligned}
$$

where

$$
C(\gamma, \beta, A, B, \alpha)=\left[2 \pi(A-B)^{2}\right]^{\frac{\gamma}{2}}(C(\lambda))^{\frac{2-\gamma}{2}}
$$

and

$$
\lambda=\frac{(1-\beta)(\mu-2)}{2-\gamma}>1
$$

Theorem 3.7. Let $f \in k-\mathcal{T}_{\mu}[A, B, C, D, \alpha]$. Then for $\mu>\frac{2-\gamma}{1-\beta}+2$,

$$
a_{n}=O(1) n^{(1-\beta)\left(\frac{\mu}{2}+1\right)+\gamma-2} \quad(n \rightarrow \infty)
$$

where $O(1)$ is a constant depending on $\gamma, \beta, A$ and $B$, and $\gamma, \beta$ are respectively given by (1.4) and (3.1).

Proof. By Cauchy Theorem, we have for $z=r e^{i \theta}$

$$
\begin{aligned}
n\left|a_{n}\right| & \leq \frac{1}{2 \pi r^{n}} \int_{0}^{2 \pi}\left|z f^{\prime}(z)\right| d \theta \\
& =\frac{1}{2 \pi r^{n}} \mathcal{L}_{r}(f) .
\end{aligned}
$$

Now, by applying Theorem 3.6 and setting $r=1-\frac{1}{n}$ as $n \rightarrow \infty$, we have the result.

Theorem 3.8. Let $f \in k-\mathcal{T}_{\mu}[A, B, C, D, \alpha]$. Then for $\mu>\frac{2-\gamma}{1-\beta}+2$,

$$
|| a_{n+1}|-| a_{n}|| \leq D(\gamma, \beta, \mu, A, B) n^{\eta-1}
$$

where $\eta=\frac{2(\gamma-\beta-1)+\mu(1-\beta)-2}{2}$ and $D(\gamma, \beta, \mu, A, B)$ is a constant that depends only on $\gamma, \beta, \mu, A, B$, and $\gamma, \beta$ are given by (1.4) and (3.1) respectively.

Proof. For $f \in k-\mathcal{T}_{\mu}[A, B, C, D, \alpha]$, we write

$$
z f^{\prime}(z)=z^{\beta}\left(s_{1}(z)\left(p_{1}(z)\right)^{\frac{\mu}{2}-1}\right)^{1-\beta}\left(h_{1}(z)\right)^{\gamma},
$$

where $s_{1} \in \mathcal{S}^{*}, p_{1} \in \mathcal{P}$ and $h_{1} \in P[A, B]$. Let $z_{1}$ be complex number with $\left|z_{1}\right|=r$. Then by Cauchy Theorem,

$$
\left|z_{1}(n+1)\right| a_{n+1}|-n| a_{n}|| \leq \frac{1}{2 \pi r^{n+1}} \int_{0}^{2 \pi}\left|z_{1}-z\right|\left|z^{\beta}\left(s_{1}(z)\left(p_{1}(z)\right)^{\frac{\mu}{2}-1}\right)^{1-\beta}\left(h_{1}(z)\right)^{\gamma}\right| .
$$


Since $s_{1} \in S^{*}$, then by a result due to Golusin [2],

$$
\left|\left(z_{1}-z\right) s_{1}\right| \leq \frac{2 r^{2}}{1-r^{2}}
$$

we have

$$
\left|z_{1}(n+1)\right| a_{n+1}|-n| a_{n}|| \leq \frac{2 r^{\beta+2}}{2 \pi r^{n+1}\left(1-r^{2}\right)} \int_{0}^{2 \pi}\left|\left(s_{1}(z)\right)^{-\beta}\left(p_{1}(z)\right)^{\left(\frac{\mu}{2}-1\right)(1-\beta)}\left(h_{1}(z)\right)^{\gamma}\right| .
$$

Now, using Distortion Theorem for starlike function $s_{1}(z)$, Holder's inequality, Corollary 3.8 and Lemma 2.3 , we have

$$
\begin{aligned}
\left|z_{1}(n+1)\right| a_{n+1}|-n| a_{n}|| \leq & \frac{2}{r^{n+1}(1-r)^{1-2 \beta}}\left(\frac{1}{2 \pi} \int_{0}^{2 \pi}\left|p_{1}(z)\right|^{\frac{(1-\beta)(\mu-2)}{2-\gamma}} d \theta\right)^{\frac{2-\gamma}{2}} \\
& \times\left(\frac{1}{2 \pi} \int_{0}^{2 \pi}\left|h_{1}(z)\right|^{2} d \theta\right)^{\frac{\gamma}{2}} \\
\leq & \frac{2}{r^{n+1}(1-r)^{1-2 \beta}}\left(\frac{1+\left[(A-B)^{2}-1\right] r^{2}}{1-r^{2}}\right)^{\frac{\gamma}{2}} \\
& \times\left(\int_{0}^{2 \pi}\left|p_{1}(z)\right|^{\frac{(1-\beta)(\mu-2)}{2-\gamma}} d \theta\right)^{\frac{2-\gamma}{2}} \\
\leq & \frac{D_{1}(\gamma, \beta, \mu, A, B)}{r^{n+1}}\left(\frac{1}{1-r}\right)^{\frac{2 \gamma-2 \beta+\mu(1-\beta)-2}{2}}
\end{aligned}
$$

where $D_{1}$ is a constant. Setting $r=1-\frac{1}{n}$ and $z_{1}=\frac{n}{n+1}$, the proof is completed.

Corollary 3.10. For $f \in k-\mathcal{T}_{2}[A, B, C, D, 0]$,

$$
|| a_{n+1}|-| a_{n}|| \leq D_{1}(\gamma, \beta, A, B) n^{(\gamma-2 \beta)-1}
$$

where $D_{1}(\gamma, \beta, A, B)$ is a constant that depends on $\gamma, \beta, A, B$.

Corollary 3.11. [15] For $f \in 0-\mathcal{T}_{\mu}[1,-1,1,-1,0]$,

$$
|| a_{n+1}|-| a_{n}|| \leq D_{1}(\mu) n^{\frac{\mu}{2}-1}
$$

where $D_{1}(\gamma, \beta, A, B)$ is a constant that depends only on $\mu$.

Theorem 3.9. $f \in k-\mathcal{T}_{\mu}[A, B, C, D, \alpha]$. maps the disk $|z|<r_{*}$ onto a convex domain, where $r_{*}$ is the smallest positive real number of the equation

$$
1-\lambda_{1} r+\lambda_{2} r^{2}+\lambda_{3} r^{3}+\lambda_{4} r^{4}=0
$$


where

$$
\begin{aligned}
& \lambda_{1}=A+B+(1-\beta) \mu+\gamma(A-B), \\
& \lambda_{2}=1+A B+(1-\beta) \mu(A+B), \\
& \lambda_{3}=(2 \beta-1)(A+B)-(1-\beta) \mu A B+\gamma(A-B), \\
& \lambda_{4}=A B(1-2 \beta),
\end{aligned}
$$

and $\gamma, \beta$ are respectively given by (1.4) and (3.1).

Proof. We set

$$
z f^{\prime}(z)=z^{\beta}\left(z \phi^{\prime}(z)\right)^{1-\beta}(h(z))^{\gamma},
$$

where $\phi \in \mathcal{V}_{\mu}$ and $h \in P[A, B]$. By logarithmic differentiation, we have

$$
\operatorname{Re} \frac{\left(z f^{\prime}(z)\right)^{\prime}}{f(z)}=\beta+(1-\beta) \operatorname{Re} \frac{\left(z \phi^{\prime}(z)\right)^{\prime}}{\phi(z)}+\gamma \operatorname{Re} \frac{z h^{\prime}(z)}{h(z)} .
$$

For $h \in P[A, B]$ and $\phi \in \mathcal{V}_{\mu}$, it is known in [6] and [22] respectively that

$$
\operatorname{Re} \frac{z h^{\prime}(z)}{h(z)} \geq-\frac{(A-B) r}{(1-A r)(1-B) r}
$$

and

$$
R e \frac{\left(z \phi^{\prime}(z)\right)^{\prime}}{\phi(z)} \geq \frac{r^{2}-\mu r+1}{1-r^{2}}
$$

Using these results, we have that

$$
\begin{aligned}
\operatorname{Re} \frac{\left(z f^{\prime}(z)\right)^{\prime}}{f(z)} \geq & {\left[\beta\left(1-r^{2}\right)(1-A r)(1-B r)+(1-\beta)\left(r^{2}-\mu r+1\right)(1-A r)(1-B r)\right.} \\
& \left.-\gamma(A-B) r\left(1-r^{2}\right)\right] /\left(1-r^{2}\right)(1-A r)(1-B r) .
\end{aligned}
$$

If we let

$$
T(r)=\beta\left(1-r^{2}\right)(1-A r)(1-B r)+(1-\beta)\left(r^{2}-\mu r+1\right)(1-A r)(1-B r)-\gamma(A-B) r\left(1-r^{2}\right) .
$$

Then

$$
T(1)=(1-\beta)(2-\mu)(1-A)(1-B) \leq 0, \quad \text { since } \mu \geq 2
$$

and

$$
T(0)=1>0 .
$$

Thus, $r \in(0,1)$. Hence, the theorem is proved.

Corollary 3.12. Let $f \in 0-\mathcal{T}_{2}[1,-1,1,-1,0]$. Then $f \in C$ for

$$
|z|<2-\sqrt{3}
$$


Corollary 3.13. Let $f \in 0-\mathcal{T}_{\mu}[1,-1,1,-1,0]$. Then $f(z)$ maps the disc

$$
|z|<\frac{1}{2}\left[\mu+2-\sqrt{\mu^{2}+4 \mu}\right]
$$

onto a convex domain

Using the well-known distortion theorems for $\phi \in \mathcal{V}_{\mu}$ and $h_{1} \in \mathcal{P}$, we prove the following.

Theorem 3.10. Let $f \in k-\mathcal{T}_{\mu}[1,-1, C, D, \alpha]$. Then

$$
A_{2}\left(a, b, c, r_{1}\right) \leq|f(z)| \leq A_{1}\left(a, b, c, r_{1}\right)
$$

where

$$
A_{1}\left(a, b, c, r_{1}\right)=\frac{2^{b-1}}{a}\left[r_{1}^{a} G\left(a, b, c,-r_{1}\right)-G(a, b, c,-1)\right]
$$

and

$$
A_{2}\left(a, b, c, r_{1}\right)=\frac{2^{b-1}}{a}\left[G(a, b, c,-1)-r_{1}^{-a} G\left(a, b, c,-r_{1}^{-1}\right)\right]
$$

where

$$
\begin{aligned}
a & =\left(\frac{\mu}{2}-1\right)(1-\beta) \gamma+1, \\
b & =2 \beta, \\
c & =\left(\frac{\mu}{2}-1\right)(1-\beta) \gamma+2,
\end{aligned}
$$

and, $\gamma$ and $\beta$ are given by (1.4) and (3.1) respectively.

Proof. Let

$$
f^{\prime}(z)=\left(\phi^{\prime}(z)\right)^{1-\beta}\left(h_{1}(z)\right)^{\gamma}, \phi \in \mathcal{V}_{\mu}, h_{1} \in \mathcal{P}
$$

Then

$$
\begin{aligned}
|f(z)| & \leq \int_{0}^{|z|}|\phi(t)|^{1-\beta}\left|h_{1}(t)\right|^{\gamma} d t \\
& \leq \int_{0}^{|z|} \frac{(1+t)^{\left(\frac{\mu}{2}-1\right)(1-\beta)}}{(1-t)^{\left(\frac{\mu}{2}+1\right)(1-\beta)}} \frac{(1+t)^{\gamma}}{(1-t)^{\gamma}} d t \\
& =\int_{0}^{|z|}\left(\frac{1+t}{1-t}\right)^{\left(\frac{\mu}{2}+1\right)(1-\beta)} \frac{(1+t)^{\gamma}}{(1-t)^{2(1-\beta)+\gamma}} \cdot d t
\end{aligned}
$$

Let $\xi=\frac{1+t}{1-t}$, then $1-t=\frac{2}{1+\xi}, d t=\frac{2}{(1+\xi)^{2}} d \xi,(1+t)=\frac{2 \xi}{1+\xi}$. Therefore,

$$
\begin{aligned}
|f(z)| & \leq 2^{2 \beta-1} \int_{1}^{\frac{1+|z|}{1-|z|}} \xi^{\left(\frac{\mu}{2}-1\right)(1-\beta)+\gamma}(1+\xi)^{-2 \beta} d \xi \\
& =2^{b-1}\left(I_{1}-I_{2}\right),
\end{aligned}
$$


where

$$
\begin{aligned}
I_{2} & =\int_{0}^{1} \xi^{a-1}(1-\xi)^{c-a-1}(1-(1 \xi))^{-b} d \xi \\
& =\frac{1}{a} G(a, b, c,-1) .
\end{aligned}
$$

To calculate $I_{1}$, let $\xi=r_{1} u$ and $r_{1}=\frac{1+r}{1-r}$. Then

$$
\begin{aligned}
I_{1} & =\int_{0}^{1}\left(r_{1} u\right)^{\left(\frac{\mu}{2}-1\right)(1-\beta)+\gamma}\left(1+r_{1} u\right)^{-2 \beta} r_{1} d u \\
& =r_{1}^{a} \int_{0}^{1} u^{a-1}\left(1+r_{1} u\right)^{-b} d u \\
& =\frac{r_{1}^{a}}{a} G\left(a, b, c,-r_{1}\right) .
\end{aligned}
$$

Using (3.10), (3.11) and (3.12), the upper bound is obvious.

Now, we proceed to calculate the lower bound. Let $d_{r}$ denotes the radius of the largest schlicht disk centered at the origin contained in the image of $|z|<r$ under the function $f(z)$. Then there exists a point $z_{0}$ with $\left|z_{0}\right|=r$, such that $|f(z)|=d_{r}$. The ray from 0 to $f(z)$ lies entirely in the image of $\Delta$ and the inverse image of this ray is a curve $\Gamma$ in $|z|<r$. Thus,

$$
\begin{aligned}
d_{r} & =\left|f\left(z_{0}\right)\right|=\int_{\Gamma}\left|f^{\prime}(z)\right|\left|d_{r}\right| \\
& =\int_{0}^{|z|}\left|\phi^{\prime}(\rho)\right|^{1-\beta}\left|h_{1}(\rho)\right|^{\gamma} d \rho \\
& \geq \int_{0}^{|z|}\left(\frac{1-\rho}{1+\rho}\right)^{\left(\frac{\mu}{2} 11\right)(1-\beta)} \frac{(1-\rho)^{\gamma}}{(1+\rho)^{2(1-\beta)+\gamma}} d \rho .
\end{aligned}
$$

Let $\nu=\frac{1-\rho}{1+\rho}$. Then $d \rho=\frac{-2}{(1+\nu)^{2}} d \nu$ and $1-\rho=\frac{2 \nu}{1+\nu}$. Going through the same process as it has been done for the upper bound, we easily obtain the lower bound and this completes the proof.

Theorem 3.11. Let $f, g \in k-\mathcal{T}_{\mu}[A, B, C, D, \alpha], \eta_{1}, c_{1}, \delta$ and $v$ be positively real, $\eta_{1}<1, c_{1}>\eta_{1}, \eta_{1}=v+\delta$. Then for the function $F(z)$ defined by

$$
\begin{gathered}
{[F(z)]^{\eta_{1}}=c_{1} z^{\eta_{1}-c_{1}} \int_{0}^{z} t^{\left(c_{1}-\delta-v\right)-1}(f(t))^{\delta}(g(t))^{v} d t} \\
\int_{\theta_{1}}^{\theta_{2}} \operatorname{Re} \frac{z F^{\prime}(z)}{F(z)}>-\pi\left[(1-\alpha)\left(\frac{C-D}{2 k+1-D}\right)\left(\frac{\mu}{2}-1\right)+\gamma\right],
\end{gathered}
$$

where $0 \leq \theta_{1}<\theta_{2} \leq 2 \pi$. 
Proof. It has been shown in [17] that the integral transformation (3.13) is a well defined analytic function in $\Delta$. Let $H(z)=\frac{z F^{\prime}(z)}{F(z)}$. Then, differentiating (3.13), we obtain

$$
F(z)^{\eta_{1}}\left[\left(c_{1}-\eta_{1}\right)+\eta_{1} H(z)\right]=c_{1} f(z)^{\delta} g(z)^{v} .
$$

Differentiating logarithmically and with some simple computations, we have

$$
H(z)+\frac{\frac{1}{\eta} z H^{\prime}(z)}{\frac{\left(c_{1}-\eta_{1}\right)}{\eta_{1}}+H(z)}=\frac{\delta}{\eta_{1}} \frac{z f^{\prime}(z)}{f(z)}+\frac{v}{\eta_{1}} \frac{z g^{\prime}(z)}{g(z)} .
$$

Now, since $f, g \in k-\mathcal{T}_{\mu}[A, B, C, D, \alpha]$, then application of Remark 3.1 gives

$$
\int_{\theta_{1}}^{\theta_{2}} \operatorname{Re}\left\{H(z)+\frac{\frac{1}{\eta} z H^{\prime}(z)}{\frac{\left(c_{1}-\eta_{1}\right)}{\eta_{1}}+H(z)}\right\}>-\pi\left[(1-\alpha)\left(\frac{C-D}{2 k+1-D}\right)\left(\frac{\mu}{2}-1\right)+\gamma\right] .
$$

On using Lemma 2.5 with $\varrho=\frac{1}{\eta}, c=c_{1}-\eta$, we complete the proof.

Theorem 3.12. Let $f, g \in k-\mathcal{T}_{\mu}[A, B, C, D, \alpha]$ and $-1<\rho \leq 0,0<\alpha_{1} \leq 1$. Then for the function $J(z)$ defined by

$$
\begin{gathered}
J(z)=\left[\left(\rho+\frac{1}{\alpha_{1}}\right) z^{1-\frac{1}{\alpha_{1}}} \int_{0}^{z} t^{\frac{1}{\alpha_{1}}-2} f(t)^{\rho} g(t) d t\right]^{\frac{1}{1+\rho}}, \\
\int_{\theta_{1}}^{\theta_{2}} \operatorname{Re} \frac{z J^{\prime}(z)}{J(z)}>-\pi\left[(1-\alpha)\left(\frac{C-D}{2 k+1-D}\right)\left(\frac{\mu}{2}-1\right)+\gamma\right],
\end{gathered}
$$

where $0 \leq \theta_{1}<\theta_{2} \leq 2 \pi$.

Proof. Set $\frac{z J^{\prime}(z)}{J(z)}=G(z)$. Then differentiating (3.14) logarithmically and with some simple calculations, we get

$$
\left(\frac{1}{\alpha_{1}}-1\right)+(\rho+1) G(z)=\frac{z^{\frac{1}{\alpha_{1}}-1} f(z)^{\rho} g(z)}{\int_{0}^{z} t^{\frac{1}{\alpha_{1}}-2} f(t)^{\rho} g(t) d t}
$$

This implies

$$
\left(\rho+\frac{1}{\alpha_{1}}\right) f(z)^{\rho} g(z)=\left(\left(\frac{1}{\alpha_{1}}-1\right)+(\rho+1) G(z)\right) J(z)^{\rho+1} .
$$

Differentiating logarithmically once again, we have

$$
H(z)+\frac{\frac{1}{1+\rho} z H^{\prime}(z)}{\frac{\left(\frac{1}{\alpha_{1}}-1\right)}{1+\rho}+H(z)}=\frac{\rho}{1+\rho} \frac{z f^{\prime}(z)}{f(z)}+\frac{1}{1+\rho} \frac{z g^{\prime}(z)}{g(z)} .
$$

Applying Lemma 2.5 with $\varrho=\frac{1}{1+\rho}, c=\frac{1}{\alpha_{1}}-1$, it follows that

$$
\int_{\theta_{1}}^{\theta_{2}} \operatorname{Re} \frac{z J^{\prime}(z)}{J(z)}>-\pi\left[(1-\alpha)\left(\frac{C-D}{2 k+1-D}\right)\left(\frac{\mu}{2}-1\right)+\gamma\right] .
$$




\section{REFERENCES}

[1] D.A. Brannan, On Functions of Bounded Boundary Rotation, Proc. Edln. Math. Soc. 2(1968/69), 330-347.

[2] G. Golusin, On distortion theorems and coefficients of univalent functions, Mat. Sb. 19(1946), 183-203 .

[3] A.W. Goodman, On close-to-convex functions of higher order, Ann. Univ. Sci. Budapest, Eötöus Sect. Math., 25(1972), 17-30

[4] A.W. Goodman, Univalent functions, Vols. I\& II, Polygonal Publishing House, Washinton, 1983.

[5] W.K. Hyman, On functions with positive real part, J. London Math. Soc. 36(1961), 34-48.

[6] W. Janowski, Some extremal problems for certain families of analytic functions, I, Ann. Polon. Math. 28 (1973), 297-326.

[7] S. Kanas and A. Wisniowska, Conic regions and k-uniform convexity, J. Comput. Appl. Math. 105(1999), 327-336.

[8] S. Kanas and A. Wisniowska, Conic domains and starlike functions, Rev. Roum. Math. Pures Appl. 45(2000), 647-657.

[9] S. Kanas, Coefficient estimates in subclasses of the Caratheodory class related to conical domain, Acta Math. Univ. Comenian, 74(2005), no. 2, 149-161.

[10] W. Kaplan, Close-to-convex Schilcht Functions, Mich. Math. J. 1 (1952), 169-185.

[11] S. Kumar and C. Ramesha, Subordination properties of uniformly convex and uniformly close to convex functions, J. Ramanujan Math. Soc. 9 (1994), no. 2, 203-214.

[12] M. Shahi, A. Muhammad and N.M. Sarfraz, Janoswski type close-to-convex functions associated with conic regions, J. Ineq. Appl. 2017,1(2017), 259.

[13] B.S. Mehrok, A Subclass of Close-to-Convex Functions, Int. J. Math. Anal, 4,(2010), no. 27, 1319-1327.

[14] K.I. Noor and M.A. Noor, Higher Order Close-to-Convex Functions related with Conic Domain, Appl. Math. Inf. Sci. $8(2014)$, no. 5, 2455.

[15] K.I. Noor, On a generalization of close-to-convexity, Internat. J. Math. Math. Sci. 6(1983), no. 2, 327-334.

[16] K.I. Noor, On subclasses of close-to-convex functions of higher order, Internat. J. Math. Math. Sci. 15(1992), no. 2, $279-290$.

[17] K.I. Noor, On a generalization of uniformly convex and related functions, Comput. Math. Appl. 61(2011), 117-125.

[18] K.I. Noor and S.N. Malik, On coefficient inequalities of functions associated with conic domains, Comput. Math. Appl. 62(2011), 2209-2217.

[19] K.I. Noor and M.A. Salim, On some classes of analytic functions, J. Inequal. Pure Appl. Math. 5(2004), no. 4, 98.

[20] F. Ronning, Uniform convex functions and a corresponding class of starlike functions, Proc. Amer. Math. Soc. 118(1993), 189-196.

[21] B. Pinchuk, A variational method for functions of bounded boundary rotation, Trans. Amer. Math. Soc. 138(1969) 107-113.

[22] B. Pinchuk, Functions with bounded boundary rotation, Isr. J. Math. 10 (1971), 716.

[23] E.M. Silvia, Subclasses of close-to-convex functions, Int. J. Math. Math. Sci. 3(1983), 449-458.

[24] K.G. Subramanian, T.V. Sudharsan and H. Silverman, On uniforly close-to-convex functions and uniformly quasiconvex functions, Int.J. Math. Math. Sci.48(2003), 3053-3058.

[25] D.K. Thomas, On starlike and close-to-convex univalent functions, J. Lond. Math. Soc. 42 (1967), 427-435. 